\title{
OBSERVATION OF TWO NEW L4 NEPTUNE TROJANS IN THE DARK ENERGY SURVEY SUPERNOVA FIELDS
}

D. W. Gerdes ${ }^{1}$, R. J. Jennings ${ }^{2}$, G. M. Bernstein ${ }^{3}$, M. Sako ${ }^{3}$, F. Adams ${ }^{1,4}$, D. Goldstein ${ }^{5,6}$, R. Kessler ${ }^{7,8}$, S. Hamilton ${ }^{1}$, T. Aвbott ${ }^{9}$, F. B. Abdalla ${ }^{10,11}$, S. Allam ${ }^{12}$, A. Benoit-Lévy ${ }^{10}$, E. Bertin ${ }^{13,14}$, D. Brooks ${ }^{10}$, E. Buckley-Geer ${ }^{12}$, D. L. Burke ${ }^{15,16}$, D. Capozzi ${ }^{17}$, A. Carnero Rosell ${ }^{18,19}$, M. Carrasco Kind ${ }^{20,21}$, J. Carretero ${ }^{22,23}$, C. E. Cunha ${ }^{15}$, C. B. D'Andrea ${ }^{17}$, L. N. DA Costa ${ }^{18,19}$, D. L. DePoy ${ }^{24}$, S. Desai ${ }^{25,26}$, J. P. Dietrich ${ }^{25,27}$, P. Doel ${ }^{10}$, T. F. Eifler ${ }^{3,28}$, A. Fausti Neto ${ }^{18}$, B. Flaugher ${ }^{12}$, J. Frieman ${ }^{12,7}$, E. Gaztanaga ${ }^{22}$, D. Gruen $^{29,27}$, R. A. Gruendi ${ }^{20,21}$, G. Gutierrez ${ }^{12}$,

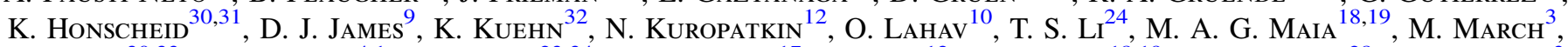
P. Martini ${ }^{30,33}$, C. J. Miller ${ }^{4,1}$, R. Miquel ${ }^{23,34}$, R. C. NiChOl $^{17}$, B. Nord ${ }^{12}$, R. Ogando ${ }^{18,19}$, A. A. Plazas ${ }^{28}$, A. K. Romer ${ }^{35}$, A. Roodman ${ }^{15,16}$, E. Sanchez ${ }^{36}$, B. Santiago ${ }^{37,18}$, M. Schubnell ${ }^{1}$, I. Sevilla-Noarbe ${ }^{35,19}$, R. C. Smith ${ }^{9}$, M. Soares-Santos ${ }^{12}$, F. Sobreira ${ }^{12,18}$, E. Suchyta ${ }^{30,31}$, M. E. C. Swanson ${ }^{21}$, G. Tarlé ${ }^{1}$, J. Thaler ${ }^{38}$, A. R. Walker ${ }^{9}$, W. WESTER ${ }^{12}$, AND Y. ZHANG ${ }^{1}$

The DES Collaboration

${ }^{1}$ Department of Physics, University of Michigan, Ann Arbor, MI 48109, USA ${ }^{2}$ Carleton College, Northfield, MN 55057, USA

${ }^{3}$ Department of Physics and Astronomy, University of Pennsylvania, Philadelphia, PA 19104, USA

${ }^{4}$ Department of Astronomy, University of Michigan, Ann Arbor, MI 48109, USA

${ }^{5}$ Department of Astronomy, University of California, Berkeley, 501 Campbell Hall, Berkeley, CA 94720, USA

${ }^{6}$ Lawrence Berkeley National Laboratory, 1 Cyclotron Road, Berkeley, CA 94720, USA

${ }^{7}$ Kavli Institute for Cosmological Physics, University of Chicago, Chicago, IL 60637, USA

${ }^{8}$ Department of Astronomy and Astrophysics, University of Chicago, 5640 South Ellis Avenue, Chicago, IL 60637, USA

${ }_{9}$ Cerro Tololo Inter-American Observatory, National Optical Astronomy Observatory, Casilla 603, La Serena, Chile

${ }^{10}$ Department of Physics \& Astronomy, University College London, Gower Street, London, WC1E 6BT, UK

${ }^{11}$ Department of Physics and Electronics, Rhodes University, P.O. Box 94, Grahamstown, 6140, South Africa

${ }_{13}^{12}$ Fermi National Accelerator Laboratory, P.O. Box 500, Batavia, IL 60510, USA

${ }^{13}$ CNRS, UMR 7095, Institut d'Astrophysique de Paris, F-75014, Paris, France

${ }^{14}$ Sorbonne Universités, UPMC Univ Paris 06, UMR 7095, Institut d'Astrophysique de Paris, F-75014, Paris, France

${ }^{15}$ Kavli Institute for Particle Astrophysics \& Cosmology, P.O. Box 2450, Stanford University, Stanford, CA 94305, USA ${ }^{16}$ SLAC National Accelerator Laboratory, Menlo Park, CA 94025, USA

${ }^{17}$ Institute of Cosmology \& Gravitation, University of Portsmouth, Portsmouth, PO1 3FX, UK

${ }^{18}$ Laboratório Interinstitucional de e-Astronomia-LIneA, Rua Gal. José Cristino 77, Rio de Janeiro, RJ-20921-400, Brazil

${ }^{19}$ Observatório Nacional, Rua Gal. José Cristino 77, Rio de Janeiro, RJ-20921-400, Brazil

${ }^{20}$ Department of Astronomy, University of Illinois, 1002 W. Green Street, Urbana, IL 61801, USA

${ }^{21}$ National Center for Supercomputing Applications, 1205 West Clark Street, Urbana, IL 61801, USA

22 Institut de Ciències de l'Espai, IEEC-CSIC, Campus UAB, Carrer de Can Magrans, s/n, E-08193 Bellaterra, Barcelona, Spain

${ }^{23}$ Institut de Física d'Altes Energies, Universitat Autònoma de Barcelona, E-08193 Bellaterra, Barcelona, Spain

${ }^{24}$ George P. and Cynthia Woods Mitchell Institute for Fundamental Physics and Astronomy, and Department of Physics and Astronomy, Texas A\&M University, College Station, TX 77843, USA

${ }^{25}$ Excellence Cluster universe, Boltzmannstrasse 2, D-85748 Garching, Germany

${ }^{26}$ Faculty of Physics, Ludwig-Maximilians University, Scheinerstrasse 1, D-81679 Munich, Germany

${ }^{27}$ Universitäts-Sternwarte, Fakultät für Physik, Ludwig-Maximilians Universität München, Scheinerstrasse 1, D-81679 München, Germany

${ }^{28}$ Jet Propulsion Laboratory, California Institute of Technology, 4800 Oak Grove Drive, Pasadena, CA 91109, USA

${ }^{29}$ Max Planck Institute for Extraterrestrial Physics, Giessenbachstrasse, D-85748 Garching, Germany

${ }^{30}$ Center for Cosmology and Astro-Particle Physics, The Ohio State University, Columbus, OH 43210, USA

31 Department of Physics, The Ohio State University, Columbus, OH 43210, USA

${ }^{32}$ Australian Astronomical Observatory, North Ryde, NSW 2113, Australia

${ }^{33}$ Department of Astronomy, The Ohio State University, Columbus, OH 43210, USA

${ }^{34}$ Institució Catalana de Recerca i Estudis Avançats, E-08010 Barcelona, Spain

${ }^{35}$ Department of Physics and Astronomy, Pevensey Building, University of Sussex, Brighton, BN1 9QH, UK

${ }^{36}$ Centro de Investigaciones Energéticas, Medioambientales y Tecnológicas (CIEMAT), Madrid, Spain

${ }^{37}$ Instituto de Física, UFRGS, Caixa Postal 15051, Porto Alegre, RS-91501-970, Brazil

${ }^{38}$ Department of Physics, University of Illinois, 1110 W. Green Street, Urbana, IL 61801, USA

Received 2015 July 18; accepted 2015 December 18; published 2016 January 28

\begin{abstract}
We report the discovery of the eighth and ninth known Trojans in stable orbits around Neptune's leading Lagrange point, L4. The objects $2014 \mathrm{QO}_{441}$ and $2014 \mathrm{QP}_{441}$ were detected in data obtained during the $2013-14$ and 2014-15 observing seasons by the Dark Energy Survey, using the Dark Energy Camera (DECam) on the 4-m Blanco telescope at Cerro Tololo Inter-American Observatory. Both are in high-inclination orbits (18.8 and 19.4, respectively). With an eccentricity of $0.104,2014 \mathrm{QO}_{441}$ has the most eccentric orbit of the 11 known stable Neptune Trojans. Here we describe the search procedure and investigate the objects' long-term dynamical stability and physical properties.
\end{abstract}

Key words: minor planets, asteroids: general 


\section{INTRODUCTION}

Trojan asteroids, together with main belt asteroids and members of the classical Kuiper Belt, constitute the only dynamically stable populations of minor planets in the Solar System. Trojans share the same orbital period as a major planet, leading or trailing this major planet by approximately 60 degrees in an orbit centered upon the L4 or L5 Lagrange point. Trojan asteroids are associated with a number of planets, including Earth (Connors et al. 2011). Jupiter has the largest and best-characterized population, with over 6000 known Trojans. The total number of $>1 \mathrm{~km}$-sized Jupiter Trojans is estimated to exceed 600,000 (Yoshida \& Nakamura 2005), comparable to the similar-sized population of the main asteroid belt (Jewitt et al. 2000). The number of large $(\gtrsim 65 \mathrm{~km})$ Neptune Trojans may exceed their Jovian counterparts by more than an order of magnitude (Chiang \& Lithwick 2005; Sheppard \& Trujillo 2006).

Since the discovery of the first Neptune Trojan, $2001 \mathrm{QR}_{322}$ (Chiang et al. 2003), only eight additional Neptune co-orbitals have been discovered prior to this work. ${ }^{39}$ Seven of the nine previously known Neptune Trojans occupy the L4 region. There is no a priori reason to expect the L4 and L5 populations to be particularly different (Sheppard \& Trujillo 2010); the dearth of known L5 Trojans can be ascribed to the fact that Neptune's L5 region is presently on a line of sight to the galactic center, a crowded field of point-like sources against which the detection of transients is observationally challenging (Sheppard \& Trujillo 2010; Parker et al. 2013).

In addition to their intrinsic interest, the population of Neptune Trojans provides an important set of constraints on the dynamical evolution of our Solar System. Although these bodies have relatively little total mass, they play the role of a canary in the coal mine: such small bodies act as test particles and are easily disrupted through a variety of channels, especially in the early epochs of Solar System history. Possible mechanisms that could affect the orbits of these bodies include gas drag in the early solar nebula (Peale 1993; Murray 1994), planetary migration (Gomes 1998; Kortenkamp et al. 2004), the growing mass of planets during their formation (Marzari \& Scholl 1998; Fleming \& Hamilton 2000), encounters with passing stars in the solar birth cluster (which can easily perturb Neptune's orbit, see Li \& Adams 2015), and a wide range of resonance phenomena (Morbidelli et al. 2005). Determining the properties and orbital characteristics of the Trojan population also places interesting constraints on theories of their formation (Chiang \& Lithwick 2005). For example, Parker (2015) uses the high mean inclination of Neptune Trojans to argue that the disk into which Neptune migrated must have been dynamically excited prior to Neptune's arrival.

In this paper, we report the discovery of the eighth and ninth known L4 Neptune Trojans, $2014 \mathrm{QO}_{441}$ and $2014 \mathrm{QP}_{441}$. This paper is organized as follows. Section 2 briefly describes the Dark Energy Survey (DES) and camera, DECam. In Section 3 we describe the supernova data reduction pipeline that is the source of transient candidates considered in this analysis, discuss our method for identifying distant solar system objects, and present our observations of the two Neptune Trojans. In Section 4 we describe numerical investigations of their long-

\footnotetext{
39 The Minor Planet Center lists a 9th object, $2004 \mathrm{KV}_{18}$, as an L5 Trojan, but with an eccentricity of 0.18 and a libration amplitude of $\sim 70^{\circ}$, it is known to be unstable on $\sim$ Myr timescales and is likely a temporarily captured scattered disk object (Nesvorný \& Dones 2002; Guan et al. 2012; Horner \& Lykawka 2012).
}

term dynamical stability. Section 5 describes their physical characteristics in the context of the broader population of Trojans. We conclude in Section 6 with a summary of our results and a discussion of their implications.

\section{THE DARK ENERGY SURVEY}

The DES (Flaugher 2005) is a five-year optical imaging survey being carried out on the 4-m Blanco telescope at Cerro Tololo Inter-American Observatory in Chile. Observations are carried out with the Dark Energy Camera (DECam; Flaugher et al. 2015), a $3 \mathrm{deg}^{2}$ prime-focus camera whose focal plane consists of $622 \mathrm{k} \times 4 \mathrm{k}$ fully depleted, red-sensitive CCDs. DECam saw first light in 2012 September, and underwent "Science Verification" operations between 2012 November and 2013 February. DECam's power as a discovery instrument for distant solar system objects was demonstrated with the discovery, during community time early in the commissioning phase, of the Sedna-like dwarf planet $2012 \mathrm{VP}_{113}$ (Trujillo \& Sheppard 2014), which has the most distant perihelion (>80 AU) of any known solar system object. Full-scale survey operations began in 2013 August and will continue through at least 2018.

To achieve the goal of measuring the dark energy equation of state to high precision, DES is divided into two distinct, interleaved surveys. The Wide Survey covers $5000 \mathrm{deg}^{2}$ of the south galactic cap in the griz $Y$ bands, imaging each survey tile approximately 10 times in each band over the course of the survey in order to perform high-statistics measurements of weak gravitational lensing, galaxy-galaxy correlations, and properties of galaxy clusters. The DES Supernova Program (DES-SN, Bernstein et al. 2012) images 10 distinct DECam fields (8 "shallow" and 2 "deep") in the griz bands at approximately weekly intervals throughout the DES observing season, which runs from mid-August through mid-February. Characteristics of these fields are shown in Table 1. Although primarily intended to find and characterize large numbers of $\mathrm{SNe}$ Ia, the supernova fields are also excellent hunting grounds for distant minor planets, which move slowly enough that they can remain in the same field of view for weeks or months at a time, and even from one DES observing season to the next. In particular, the two Stripe-82 fields ("S-fields") and the three XMM-LSS fields ("X fields") have moderate ecliptic latitudes ranging from $-20^{\circ}$ to $-15^{\circ}$, and also, serendipitously, lead the present position of Neptune by approximately $60^{\circ}$ of ecliptic longitude. We search for Neptune Trojans in these fields.

\section{OBSERVATIONS AND SEARCH STRATEGY}

This analysis uses imaging data processed for the Supernova Survey by the DES data management (DESDM) pipeline (Desai et al. 2012; Mohr et al. 2012) at the National Center for Supercomputing Applications. After cleanup and detrending, astrometric solutions are obtained using SCAMP (Bertin et al. 2006), with stellar positions in each image matched to the UCAC4 catalog (Zacharias et al. 2013) to an absolute astrometric precision of roughly 0 ". 1 . Image subtraction is performed with the HOTPANTS algorithm (Becker 2015) using point-spread function (PSF)-matched deep templates obtained during Science Verification. Source detection is performed on the subtracted images using Sextractor (Bertin \& Arnouts 1996). A machine-learning algorithm (Goldstein et al. 2015) reduces artifacts from instrumental or image- 
Table 1

Summary of DES Supernova Fields

\begin{tabular}{|c|c|c|c|c|c|}
\hline \multirow[b]{2}{*}{ Field } & \multirow[b]{2}{*}{ Depth } & \multicolumn{2}{|c|}{ Center (Equatorial) } & \multicolumn{2}{|c|}{ Center (Ecliptic) } \\
\hline & & R.A. (hr) & decl. (deg) & $\lambda(\operatorname{deg})$ & $\beta(\operatorname{deg})$ \\
\hline $\mathrm{C} 1$ & shallow & 03:37:05.83 & $-27: 06: 41.8$ & $42: 49: 30.2$ & $-44: 52: 29.6$ \\
\hline $\mathrm{C} 2$ & shallow & 03:37:05.83 & $-29: 05: 18.2$ & $41: 52: 47.4$ & $-46: 44: 19.2$ \\
\hline $\mathrm{C} 3$ & deep & $03: 30: 35.62$ & $-28: 06: 00.0$ & $40: 25: 56.8$ & $-45: 19: 21.4$ \\
\hline $\mathrm{X} 1$ & shallow & $02: 17: 54.17$ & $-04: 55: 46.2$ & $30: 28: 17.9$ & $-17: 38: 53.3$ \\
\hline $\mathrm{X} 2$ & shallow & $02: 22: 39.48$ & $-06: 24: 43.6$ & 31:06:23.4 & $-19: 26: 48.6$ \\
\hline $\mathrm{X} 3$ & deep & $02: 25: 48.00$ & $-04: 36: 00.0$ & 32:31:58.0 & $-18: 00: 28.3$ \\
\hline S1 & shallow & 02:51:16.80 & 00:00:00.0 & $40: 22: 15.4$ & $-15: 41: 10.2$ \\
\hline S2 & shallow & $02: 44: 46.66$ & $-00: 59: 18.2$ & $38: 26: 48.0$ & $-16: 07: 37.7$ \\
\hline E1 & shallow & $00: 31: 29.86$ & $-43: 00: 34.6$ & $346: 05: 17.0$ & $-41: 44: 05.3$ \\
\hline E2 & shallow & 00:38:00.00 & $-43: 59: 52.8$ & $346: 43: 33.9$ & $-43: 11: 58.4$ \\
\hline
\end{tabular}

subtraction effects by a factor of nine. All data have been reprocessed using the difference-imaging pipeline described in Kessler et al. (2015). The efficiency of the pipeline for reconstructing and accepting true point-like sources is measured by inserting fake supernovae into the images using the SNANA code (Kessler et al. 2009) prior to image-subtraction. The efficiency reaches $50 \%$ for signal-to-noise ratio $(\mathrm{S} / \mathrm{N})$ of about 5 (see Figure 7 in Kessler et al. 2015), which corresponds to an average $r$-band magnitude depth of 23.5 in the shallow fields and 24.5 in the deep fields. We compute PSF magnitudes in the $A B$ system. Images are calibrated nightly to tertiary standards to a photometric precision of $2 \%$ on average.

The $r$ and $i$ bands have the best $\mathrm{S} / \mathrm{N}$, with $5 \sigma$ limiting magnitudes of 23.8 in the shallow fields and 24.5 in the deep fields. We therefore carry out the initial candidate search in these bands, and add observations from the $g$ and $z$ bands once candidates have been identified. Our target fields contain approximately 185,000 single-epoch transients in the $r i$ bands from the first (2013 August-2014 February) and second (2014 August-2015 February) DES observing seasons.

An object at 30 AU undergoing retrograde motion moves at an apparent rate of up to $4^{\prime \prime}$ per hour. In the shallow fields, exposure times range from $150 \mathrm{~s}$ in the $r$ band to $400 \mathrm{~s}$ in the $z$ band. These exposures are sufficiently short that such an object appears stellar and is accepted with high efficiency by the machine-learning cut. In the deep fields, however, image subtraction and source detection are ordinarily performed on co-added sequences that range from $3 \times 200 \mathrm{~s}$ in the $g$ band to $11 \times 360 \mathrm{~s}$ in the $z$ band. Because such a procedure would frequently result in the rejection of slow-moving transients, for the deep fields we have also run a modified version of the transient detection pipeline that uses the single-epoch exposures, while reducing the magnitude depth to 24.2. This procedure has the added advantage of producing a fine-grained time series well-suited for light-curve measurements as discussed in Section 5.

The apparent motion of a distant Solar System object in the roughly one week between DES visits to each field is due largely to Earth's reflex motion. Beginning with the list of transients identified in a given visit by the difference-imaging pipeline, we search for counterparts at two subsequent visits in a window consistent with seasonally appropriate reflex motion at a rate of less than $150^{\prime \prime} /$ day. Triplets selected via this geometrical technique are tested for goodness of fit to an orbit using code derived from the work of (Bernstein \& Khushalani 2000, henceforward BK) by requiring $\chi^{2} /$ dof $<2$.

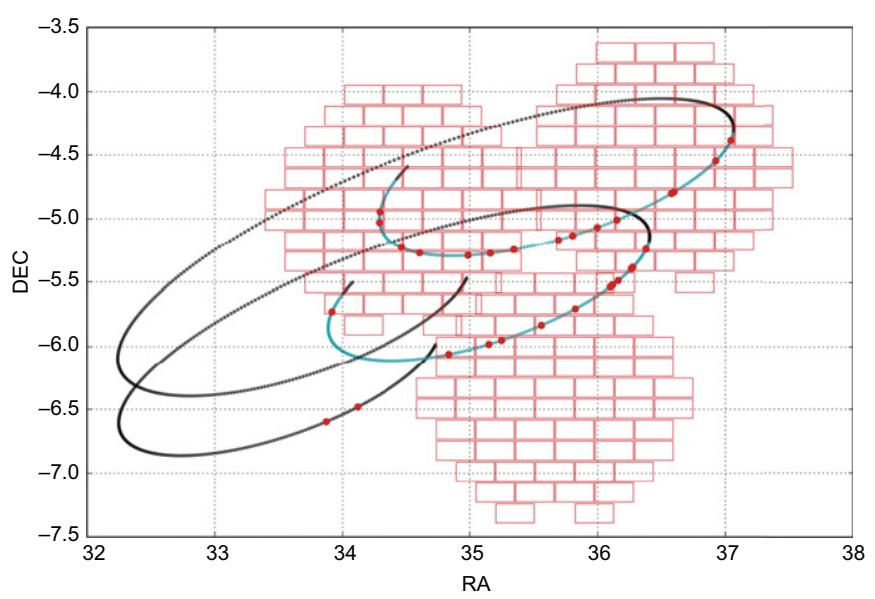

Figure 1. Observed trajectories of $2014 \mathrm{QO}_{441}$ (lower curve) and $2014 \mathrm{QP}_{441}$ (upper curve) relative to the three DES XMM-LSS supernova fields over the first two DES observing seasons. The cyan portions of the trajectories encompass the 2014-15 DES observing season. Large dots indicate nights on which the objects were detected. Each rectangle represents one DECam CCD. $2014 \mathrm{QO}_{441}$ was also detected in wide-survey exposures outside of the supernova fields on two nights in 2013 October.

Observations from other bands and visits are added iteratively, subject to this same requirement. For candidates consisting of multiple observations over four or more nights, the combinatoric background is negligible.

$2014 \mathrm{QO}_{441}$ and $2014 \mathrm{QP}_{441}$ were initially detected at apparent $r$-band magnitudes of 23.3 and 23.9 in the deep XMM-LSS field on 2014 August 21, at ecliptic latitudes of -18.5 and $-18^{\circ} .0$, respectively. $2014 \mathrm{QO}_{441}$ was observed 69 times on 12 different nights through 2015 January 29, while $2014 \mathrm{QP}_{441}$ was observed 62 times on 13 nights through 2015 January 22. In addition, $2014 \mathrm{QO}_{441}$ was recovered in five 90second wide-survey exposures from 2013 October 2 to 13. The trajectories of these two objects are shown in Figure 1. Orbital elements and other properties of these two objects are summarized in Table 2. The 2013 observations of $2014 \mathrm{QO}_{441}$ increase its arc length by nearly a year and substantially reduce the uncertainty in the orbit. A summary of these objects' properties in comparison with the other known Neptune Trojans is shown in Table 3.

\section{DYNAMICAL STABILITY}

To investigate the long-term behavior of the orbits, we have simulated swarms of 1000 clones each of $2014 \mathrm{QO}_{441}$ and 
Table 2

Orbital Elements and Other Properties of the Two New Trojans

\begin{tabular}{lll}
\hline \hline Parameter & $2014 \mathrm{QO}_{441}$ & $2014 \mathrm{QP}_{441}$ \\
\hline$a(\mathrm{AU})$ & $30.102 \pm 0.001$ & $30.108 \pm 0.011$ \\
$e$ & $0.1046 \pm 0.0004$ & $0.067 \pm 0.002$ \\
$i(\mathrm{deg})$ & $18.8316 \pm 0.0003$ & $19.405 \pm 0.001$ \\
$\omega(\mathrm{deg})$ & $113.1 \pm 1.0$ & $1.74 \pm 0.67$ \\
$\Omega(\mathrm{deg})$ & $106.998 \pm 0.004$ & $96.538 \pm 0.007$ \\
Perihelion date & $1937 / 11 / 28 \pm 176 \mathrm{~d}$ & $2043 / 4 / 16 \pm 79 \mathrm{~d}$ \\
Epoch JD & 2457000.5 & 2457000.5 \\
Libration period (year) & $9074 \pm 3$ & $9114 \pm 5$ \\
Libration amplitude (deg) & $11.1 \pm 0.1$ & $6.0 \pm 1.9$ \\
Arc length (days) & 484 & 154 \\
Apparent mag (r) & 23.3 & 23.9 \\
$H_{V}$ & 8.2 & 9.2 \\
$r-i(\mathrm{mag})$ & $0.21 \pm 0.10$ & $0.20 \pm 0.06$ \\
$i-z(\mathrm{mag})$ & $0.01 \pm 0.11$ & $0.08 \pm 0.08$ \\
Diameter (km) & 135 & 85 \\
\end{tabular}

Note.

${ }^{\mathrm{a}}$ Assuming 5\% albedo.

$2014 \mathrm{QP}_{441}$. The initial orbital elements of each clone were drawn from a multivariate normal distribution using the full six-dimensional covariance matrix obtained from fitting the observations using the BK code. We followed each clone for $10 \mathrm{Myr}$ in the presence of the four giant planets using the hybrid symplectic algorithm in the MERCURY6 $N$-body integrator (Chambers 1999). Each of the clones remained in a bound orbit about L4, evolving stably in aei-space. An example is shown in Figure 2.

To compute the libration amplitudes and periods, we follow the procedure of Parker et al. (2013) and Parker (2015). Using the same 1000 clones of each object, we define the half-peak rms libration amplitude for each clone to be

$$
L_{11}=\left(\frac{2}{N} \sum_{i=1}^{N}\left(\phi_{i}-\langle\phi\rangle\right)^{2}\right)^{1 / 2},
$$

where $i$ runs over the $N$ samples of each clone during the $10 \mathrm{Myr}$ integration. The resonant argument $\phi_{11}=\lambda_{N}-\lambda_{T}$, where $\lambda_{N}$ and $\lambda_{T}$ are the mean longitude of Neptune and the Trojan, respectively (Chiang et al. 2003). The mean longitude computed at each epoch of the integration is

$$
\lambda=M+\Omega+\omega,
$$

where $M$ is the mean anomaly, $\Omega$ is the longitude of the ascending node, and $\omega$ is the argument of perihelion. Libration periods are obtained from a spectral decomposition of $\phi(t)$, and errors are obtained from the rms scatter among the clones. We find that $2014 \mathrm{QO}_{441}$ librates with an rms amplitude of $11^{\circ} .1 \pm 0.1$ and a period of $9074 \pm 3$ years, while for $2014 \mathrm{QP}_{441}$ we find a libration amplitude of $6.0 \pm 1.9$ and a period of $9114 \pm 5$ years. The orbital motion of both Trojans in the co-rotating Neptunian frame over the course of one full libration period is shown in Figure 3.

In addition to the $\sim 9100$-year libration periods, we observe oscillations in eccentricity and inclination on 1-2 Myr timescales, which are a by-product of the long-term exchange of angular momentum between Jupiter and Saturn. To investigate these further we have continued 32 of the integrations for each object for 1 Gyr. We observe that these oscillations continue, but do not grow in amplitude, over the full timespan of the integrations for $2014 \mathrm{QP}_{441}$. Five of the 32 clones of $2014 \mathrm{QO}_{441}$ did not survive the full $1 \mathrm{Gyr}$ integration, with the earliest instability occurring between 500 and $600 \mathrm{Myr}$. Thus, based on our current knowledge of their orbits, $2014 \mathrm{QP}_{441}$ is apparently stable in its present configuration, while $2014 \mathrm{QO}_{441}$ possesses a dynamical half-life on the order of 4 Gyr. Both objects could therefore be primordial members of the disk into which Neptune migrated.

\section{PHYSICAL CHARACTERIZATION}

The measured $r-i$ and $i-z$ colors shown in Table 2 indicate that both objects are slightly red. The derived $V-R$ magnitudes of $\approx 0.44 \pm 0.11$, obtained using the transformation equations in Smith et al. (2002), are consistent with previously reported colors of the L4 and L5 Neptune Trojans (Parker et al. 2013) as well as Jupiter Trojans and neutral Centaurs, and are slightly bluer than classical KBOs. This similarity may indicate a common formation mechanism and history, although future near-infrared photometry may reveal otherwise unapparent population differences as is known to be the case with Jupiter Trojans (Wong et al. 2014).

We examined the light curves of each object to search for periodic behavior indicative of rotation or a possible binary system. We carried out this analysis using both the Phase Dispersion Minimization (Stellingwerf 1978) and generalized Lomb-Scargle periodogram (Scargle 1982; Zechmeister \& Kürster 2009) techniques. We found no evidence for modulations on timescales between 0.5 and $48 \mathrm{hr}$, leading us to conclude that within our photometric sensitivity both $2014 \mathrm{QO}_{441}$ and $2014 \mathrm{QP}_{441}$ are relatively round and featureless.

\section{SUMMARY}

This paper reports the discovery of $2014 \mathrm{QO}_{441}$ and $2014 \mathrm{QP}_{441}$ which represent the eighth and ninth members of the L4 family of Neptune Trojans. They appear to be dynamically stable on $>$ Gyr timescales and have colors similar to other Trojans. Their high inclinations lend further support to the idea that Neptune Trojans are a dynamically "hot" population that may have been excited prior to capture by Neptune (Parker 2015). The Dark Energy Survey will have further opportunities to observe $2014 \mathrm{QO}_{441}$ during the 2015-16 and 2016-17 seasons, when it will again enter the deep XMM-LSS supernova field. $2014 \mathrm{QP}_{441}$ will also be visible in this field during the 2015-16 season, then will briefly pass through one of the Stripe-82 fields during the 2016-17 and 2017-18 seasons. Further measurements of this object will improve the estimate of its dynamical lifetime.

The existence of the Neptune Trojans, along with other small bodies in the Solar System, poses a set of interesting dynamical problems. These bodies could have been placed in their current orbits at the epoch of solar system formation, or perhaps more recently. In the former scenario, the orbits must be stable over the $\sim 4.6$ Gyr age of the Sun. Sufficiently large perturbations to the orbit of Neptune, or the other giant planets, could lead to the removal of Trojans from their librations about the Lagrange points (e.g., Figure 2 shows that small perturbations of Neptune due to the other planets lead to changes in the resonant angle). As a result, the long-term stability of these orbits implies upper limits on the degree of disruption suffered by the solar system. 
Table 3

Properties of Neptune Trojans

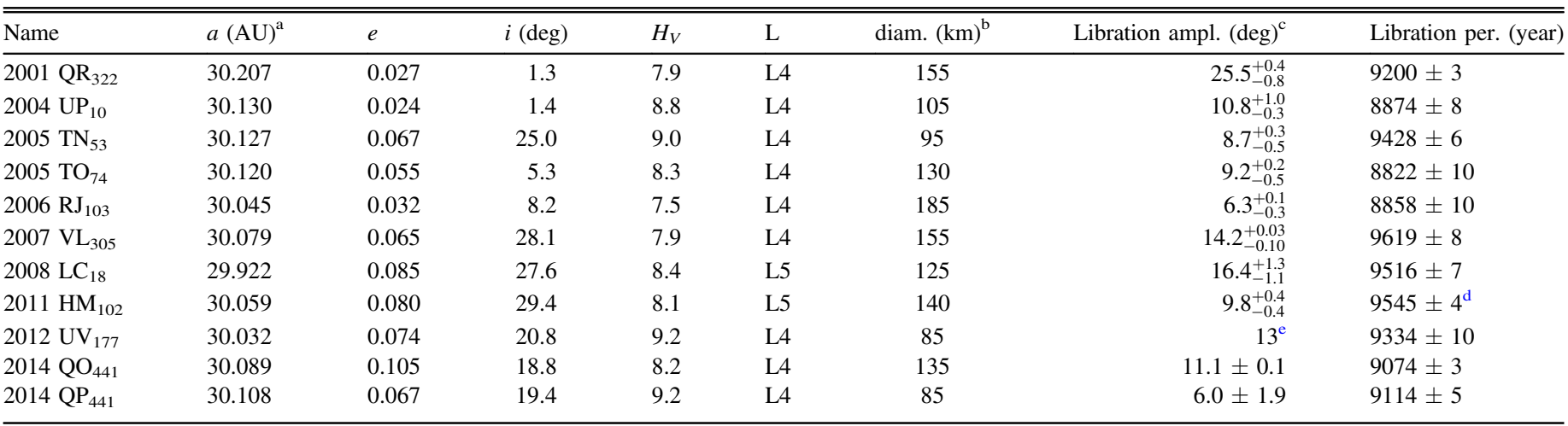

Notes.

a Barycentric osculating elements at epoch 2014 December 09.

b Assuming 5\% albedo.

${ }^{c}$ Values for first 8 objects from Parker (2015).

${ }^{\mathrm{d}}$ Parker et al. (2013).

${ }^{\mathrm{e}}$ Alexandersen et al. (2014).
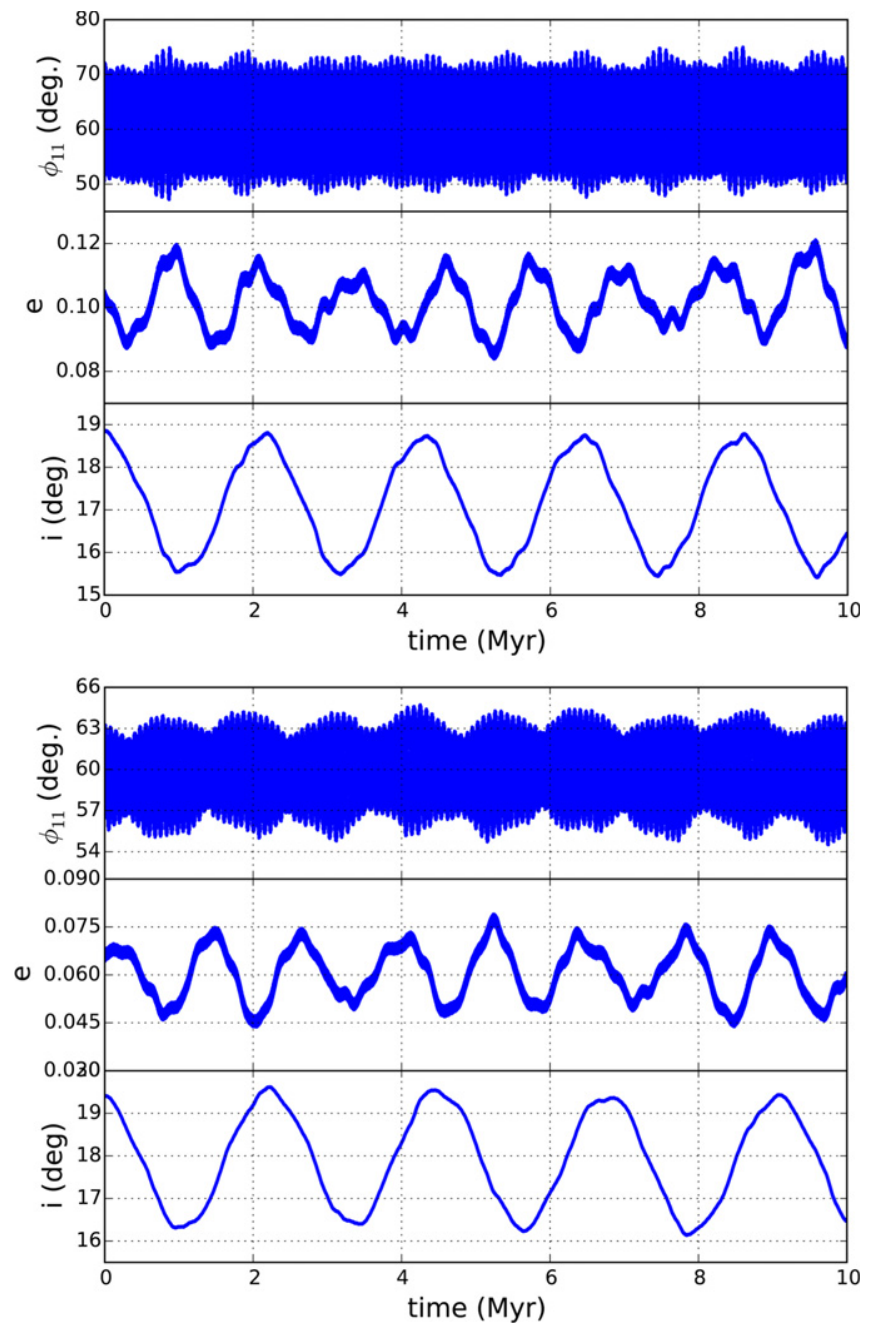

Figure 2. Evolution of the resonant angle $\phi_{11}$ and the orbital parameters $e$ and $i$ over a $10 \mathrm{Myr}$ integration for the best-fit orbits of $2014 \mathrm{QO}_{441}$ (top) and $2014 \mathrm{QP}_{441}$ (bottom). This pattern continues stably for each of the 1000 clones analyzed.

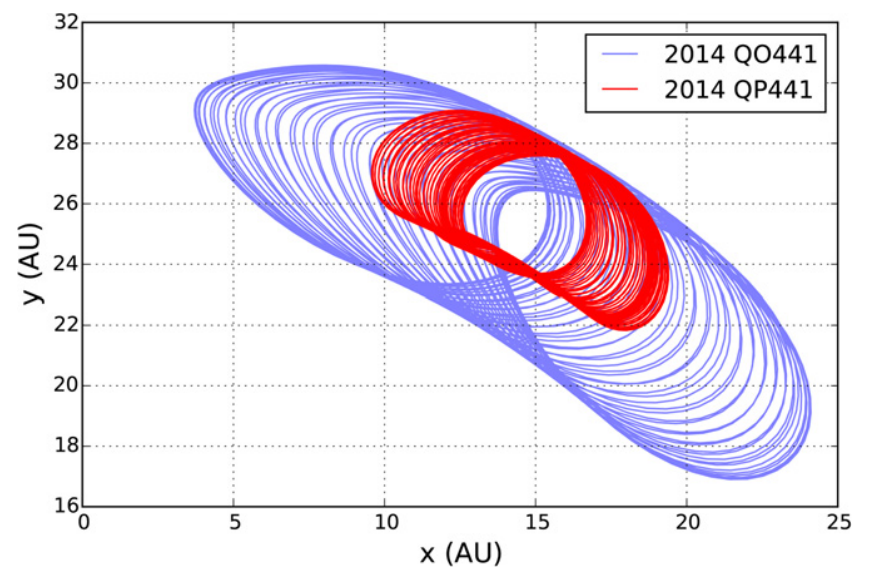

Figure 3. Orbital motion of $2014 \mathrm{QO}_{441}$ (blue) and $2014 \mathrm{QP}_{441}$ (red) in the ecliptic plane over the course of one full $\sim 9100$-year libration period as seen from a frame rotating counterclockwise at the speed of Neptune. In this coordinate system the Sun is at $(0,0)$ and Neptune lies along the $x$-axis between $x=28.9107$ and $x=30.3271$. Each individual sub-cycle of the full pattern corresponds to one 165 -year orbit.

On the other hand, if the placement of the Trojans into their currently observed orbits is more recent, then the mechanism that populates such orbits must be understood. These issues thus present a rich set of open dynamical questions.

Although this work has emphasized the detailed study of these two new Neptune Trojans, an additional 27 Kuiper Belt and other trans-Neptunian objects discovered in our search of the DES supernova fields have been given provisional designations by the Minor Planet Center. The most notable of these is $2013 \mathrm{RF}_{98}$, which has a semimajor axis of $325 \mathrm{AU}$ and a perihelion of $36 \mathrm{AU}$, making it one of the longest-period trans-Neptunians known. This analysis will be described in forthcoming work.

With three years remaining in the Dark Energy Survey, we can confidently anticipate that several dozen new transNeptunian objects, perhaps including more Neptune Trojans, will enter the supernova fields and be discovered by the 
techniques described in this paper. But the supernova fields represent less than $1 \%$ of the full $5000 \mathrm{sq}$. deg. wide survey area. Although the sparse cadence with which a given wide survey region is observed-typically 2-4 times per season in each of five filters-makes the identification of slow-moving transients more challenging, a search in the wide survey fields is likely to result in of the order of 100 new TNOs to a limiting magnitude of $r \sim 23.8$. Moreover, the wide survey footprint includes a small region near the ecliptic, but is distributed primarily over higher ecliptic latitudes, an area not well covered by previous TNO searches. This makes DES particularly well suited for the detection of the higherinclination, "hot" TNO populations. Our preliminary studies indicate that DES's combination of area and depth should result in at least five times more such objects than any previous survey. Because the detached/inner Oort cloud population is even less likely to be confined to the ecliptic region, we expect DES to have relatively higher sensitivity to this very interesting and rare group of objects compared to prior searches, shedding further light on the processes that shaped the Solar System.

We are grateful for the extraordinary contributions of our CTIO colleagues and the DES Camera, Commissioning and Science Verification teams for achieving excellent instrument and telescope conditions that have made this work possible. The success of this project also relies critically on the expertise and dedication of the DES Data Management organization. Funding for the DES Projects has been provided by the U.S. Department of Energy, the U.S. National Science Foundation, the Ministry of Science and Education of Spain, the Science and Technology Facilities Council of the United Kingdom, the Higher Education Funding Council for England, the National Center for Supercomputing Applications at the University of Illinois at Urbana-Champaign, the Kavli Institute of Cosmological Physics at the University of Chicago, Financiadora de Estudos e Projetos, Fundação Carlos Chagas Filho de Amparo à Pesquisa do Estado do Rio de Janeiro, Conselho Nacional de Desenvolvimento Científico e Tecnológico and the Ministério da Ciência e Tecnologia, the Deutsche Forschungsgemeinschaft, and the Collaborating Institutions in the Dark Energy Survey.

The Collaborating Institutions are Argonne National Laboratory, the University of California at Santa Cruz, the University of Cambridge, Centro de Investigaciones Energeticas, Medioambientales y Tecnologicas-Madrid, the University of Chicago, University College London, the DES-Brazil Consortium, the Eidgenössische Technische Hochschule (ETH) Zürich, Fermi National Accelerator Laboratory, the University of Edinburgh, the University of Illinois at Urbana-Champaign, the Institut de Ciencies de l'Espai (IEEC/CSIC), the Institut de
Fisica d'Altes Energies, Lawrence Berkeley National Laboratory, the Ludwig-Maximilians Universität and the associated Excellence Cluster universe, the University of Michigan, the National Optical Astronomy Observatory, the University of Nottingham, The Ohio State University, the University of Pennsylvania, the University of Portsmouth, SLAC National Accelerator Laboratory, Stanford University, the University of Sussex, and Texas A\&M University.

This paper has gone through internal review by the DES collaboration.

\section{REFERENCES}

Alexandersen, M., Gladman, B., Kavelaars, J. J., et al. 2014, arXiv:1411.7953 Becker, A. 2015, ascl soft: 1504.004

Bernstein, G., \& Khushalani, B. 2000, AJ, 120, 3323

Bernstein, J. P., Kessler, R., Kuhlmann, S., et al. 2012, ApJ, 753, 152

Bertin, E. 2006, in ASP Conf. Ser. 351, Astronomical Data Analysis Software and Systems XV, ed. C. Gabriel et al. (San Francisco, CA: ASP), 112

Bertin, E., \& Arnouts, S. 1996, A\&AS, 117, 393

Chambers, J. E. 1999, MNRAS, 304, 793

Chiang, E. I., Jordan, A. B., Millis, R. L., et al. 2003, AJ, 126, 430

Chiang, E. I., \& Lithwick, Y. 2005, ApJ, 628, 520

Connors, M., Wiegert, P., \& Veillet, C. 2011, Natur, 475, 481

Desai, S., Armstrong, R., Mohr, J. J., et al. 2012, ApJ, 757, 83

Flaugher, B. 2005, IJMPA, 20, 3121

Flaugher, B., Diehl, H. T., Honscheid, K., et al. 2015, AJ, 150, 150

Fleming, H. J., \& Hamilton, D. P. 2000, Icar, 148, 479

Goldstein, D. A., DAndrea, C. B., Fischer, J. A., et al. 2015, AJ, 150, 82

Gomes, R. S. 1998, AJ, 116, 2590

Guan, P., Zhou, L.-Y., \& Li, J. 2012, RAA, 12, 1549

Horner, J., \& Lykawka, P. S. 2012, MNRAS, 426, 159

Jewitt, D. C., Trujillo, C. A., \& Luu, J. X. 2000, AJ, 120, 1140

Kessler, R., Bernstein, J. P., Cinabro, D., et al. 2009, PASP, 121, 1028

Kessler, R., Marriner, J., Childress, M., et al. 2015, AJ, 150, 172

Kortenkamp, S. J., Malhotra, R., \& Michtchenko, T. 2004, Icar, 167, 347

Li, G., \& Adams, F. C. 2015, MNRAS, 448, 344

Marzari, F., \& Scholl, H. 1998, Icar, 131, 41

Mohr, J. J., Armstrong, R., Bertin, E., et al. 2012, Proc. SPIE, 8451, 0

Morbidelli, A., Levison, H. F., Tsiganis, K., \& Gomes, R. 2005, Natur, 435,462

Murray, C. D. 1994, Icar, 112, 465

Nesvorný, D., \& Dones, L. 2002, Icar, 160, 271

Parker, A. H. 2015, Icar, 247, 112

Parker, A. H., Buie, M. W., Osip, D. J., et al. 2013, AJ, 145, 96

Peale, S. J. 1993, Icar, 106, 308

Scargle, J. D. 1982, ApJ, 263, 835

Sheppard, S. S., \& Trujillo, C. A. 2006, Sci, 313, 511

Sheppard, S. S., \& Trujillo, C. A. 2010, Sci, 329, 1304

Smith, J. A., Tucker, D. L., Kent, S., et al. 2002, AJ, 123, 2121

Stellingwerf, R. F. 1978, ApJ, 224, 953

Trujillo, C. A., \& Sheppard, S. S. 2014, Natur, 507, 471

Wong, I., Brown, M. E., \& Emery, J. P. 2014, AJ, 148, 112

Yoshida, F., \& Nakamura, T. 2005, AJ, 130, 2900

Zacharias, N., Finch, C. T., Girard, T. M., et al. 2013, AJ, 145, 44

Zechmeister, M., \& Kürster, M. 2009, A\&A, 496, 577 